\title{
Impact Analysis of the Valve to the Limitation Characters of Reciprocating Compressor
}

\author{
Zhang Si-yang \\ Harbin Institute of Technology Astronautics Institute \\ Harbin China \\ z90sy@163.com
}

\begin{abstract}
Valves play an important role in the reciprocating compressor running, which will open and close according to the different piston work phases in the reciprocating compressor to control the gas discharge or suction. The time to open and close of the valves have limited with many affection which affects the improvement of the characters of a compressor. The thesis analyses the affections about restrict the characters through building the kinematics and mechanics equation and calculating the whole process time. Then some advices are given to improve the performances of the compressor.
\end{abstract}

Keywords- Top-speed; Valve; Resultant force; Mechanical equation; Kinetic equation; Limi tion character

\section{INTRODUCTION}

Reciprocating compressors are one of the most popular machines in the world. The effective and possible models which discharge more gas and increase more pressure are required to help in both reducing production costs and increasing its efficiency. For these requirements, lots of research work has been conducted with state of the art technologies in decreasing the size and increasing its characters in reciprocating compressors. To develop more accurate methods, many investigations have been carried out on developing mathematic models of compressors. Costagliola ${ }^{[1]}$ built the first reciprocating compressor mathematical model. In this model the main concern was the dynamics of the valves. Wambasganss ${ }^{[2]}$ developed with a similar model, a high-speed hermetic compressor with reed valves, who paid more attention to the dynamics of the valves in which several degrees of freedom were allowed. M. Elhaj, et al ${ }^{[3 \sim 6]}$ developed the valve model in which the conditions and force analysis were thought more carefully. In the working process of the reciprocating compressor the pressure produced through the motion of the reciprocate compressor piston and the gas was transferred through the suction and discharge valve. With the development of the compressor higher pressure and more flow gas are needed to improve the modern production, so improve the running speed of the units of the compressor is the important measure to improve the compressor performance, such as accelerating the crank which can increase the compression time and exhaust more gas. But the speed can't accelerate infinitely. And many components will affect the compressor's characters

\author{
Xu Min-qiang \\ Harbin Institute of Technology Astronautics Institute \\ Harbin China
}

such as the dimension of the piston, the speed of the crank, the thickness of the cylinder and the valve. The thesis according to the mechanical and kinetic analysis of the valve seeks the reason of restricting improving the performance of the compressor and gives some advices to improving the performance.

\section{MOdEL ANALysis OF THE COMPRESSOR VALVE}

The main motivation of the valve depends on the strain from the energy that bumps plate and lifter limiter when the valve disc was opened and closed, whose energy has relation with the thrust that the gas push the disc, the thickness, the lifting buffer ability, the circle speed, the temperature in the cylinder pressure, and so on. The force analysis of suction valve includes elastic force, gas current thrust, inertial force of the disc, gravity of the plate, gravity of the spring, viscous force of the liquid and other force can ignored. In addition to above forces the discharge valve includes elastic force of the gas, which is internal force. The motive characters of the valve can be expressed through the inertial force. The vibration of the valve disc can be simplified only by the gas current thrust. Mechanical equation can be given at the example of the suction valve.

\section{A. Analysis of the Elastic Force}

The force analysis of the valve can include two parts: the valve plate can be first take on as elastic thin plate and then take on as stiff thin plate. As the plate disc is thin very much and meets the condition of $1 / 80 \leqslant \delta / \mathrm{b} \leqslant 0.5$, so the plate that meets the Kirchhoff three hypothesizes is shown in figure 1.

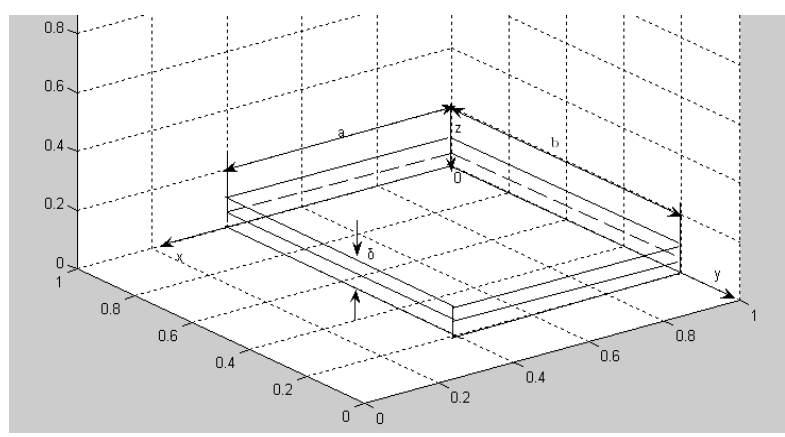

Figure 1. The diagrammatic sketch 
The direct line perpendicular to the central surface still keeps direct after deformation and keeps the same length. The stress components $\sigma_{z}, \tau_{z x}, \tau_{z y}$ Perpendicular to the central surface far less than other stress components, whose deformation can ignore, are necessary for the balance. The every point in the central surface has the displacement $\mathrm{w}$ perpendiculars the central surface without displacement parallel the central surface. So

$$
u_{z=0}=0, \quad v_{z=0}=0, \quad w=w(x, y)
$$

The thin plate kinetic equation:

$$
\begin{gathered}
\frac{\partial^{4} w}{\partial x^{4}}+2 \frac{\partial^{4} w}{\partial x^{2} \partial y^{2}}+\frac{\partial^{4} w}{\partial y^{4}}=\frac{q}{D} \\
D=\frac{-E \delta^{3}}{12\left(1-v^{2}\right)}
\end{gathered}
$$

Where

$$
\begin{gathered}
q=D \nabla^{2} \nabla^{2} w \\
q=\frac{-E \delta^{3}}{12\left(1-v^{2}\right)}\left(\frac{\partial^{4} w}{\partial x^{4}}+2 \frac{\partial^{4} w}{\partial x^{2} \partial y^{2}}+\frac{\partial^{4} w}{\partial y^{4}}\right)
\end{gathered}
$$

\section{B. Analysis of the Gas Current Thrust}

The current resultant force through the different inner and outer pressure takes on the plate which overcomes the spring force and inertia force. The current force depends on the gas pressure and work area of the valve disc, so $F_{\beta}=\frac{d p}{d t} S$

\section{Analysis of the Gas Spring Force}

When the valve opens and closes as the reasons of the relative motion same as to press the gas which restricts the motion of the valve disc if the gas is closed. The phenomena can take as a gas spring whose model should be discussed for the leakage of the gas from the other direction.

We can establish the gas spring force model. As shown in figure 2 . The gas spring suffers the force $F_{f}$. We can think that the area volume and leakage coefficient are the function of the pressure and spring deform $\mathrm{x}$, we can get the equation:

$$
\left(p_{0}+p_{a}\right) v_{0}^{\alpha}=\left(p_{0}+p_{b}\right) v^{\alpha} l
$$

Where, $l$ is the leakage coefficient.

$$
\begin{gathered}
F=\frac{1}{l}\left(\frac{V_{0}}{V}\right)^{\alpha}\left[\left(p_{0}+p_{a}\right)-p_{0}\right] \\
k=\frac{d F}{d x}=-\frac{1}{l^{2}}\left(\left(\frac{V_{0}}{V}\right)^{\alpha}\left[\left(p_{0}+p_{a}\right)-p_{0}\right]\right) s \frac{d l}{d x}- \\
\frac{n}{l}\left(p_{0}+p_{a}\right) s\left(\frac{V_{0}}{V}\right)^{\alpha} \frac{d V}{d x}+\frac{1}{l}\left(\frac{V_{0}}{V}\right)^{\alpha}\left[\left(p_{0}+p_{a}\right)-p_{0}\right] \frac{d s}{d x}
\end{gathered}
$$

From the equation 8 we can know the $\mathrm{k}$ is related with three items we can suppose k1 k2、 k3. So:

$$
\mathrm{K}=\mathrm{k} 1+\mathrm{k} 2+\mathrm{k} 3
$$

$\mathrm{F}_{\mathrm{f}}$

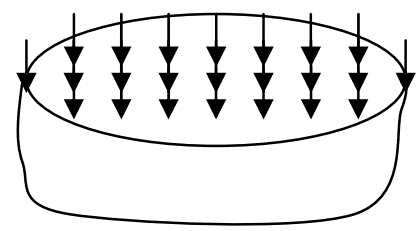

Figure 2. Gas spring model

\section{Analysis of the Resultant Force}

As the figure 3.shown, the valve will suffer less force when it is opened, but it will suffer more force when it is closed which is the action of inertia, gas force and spring force.
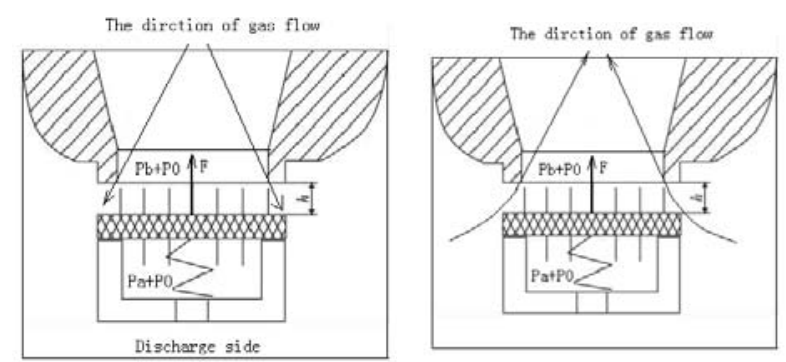

Figure 3. Force analysis of suction and discharge valve

The resultant force on the valve is from three forces: weight of valve $\mathrm{w}$, the preset spring force Fs and the force $\left(\mathrm{P}_{\mathrm{b}}-\mathrm{P}_{\mathrm{a}}\right)$ due to pressure difference between each side of the valve.

The mechanical equation:

$\Sigma F=-W+F_{S}-C_{f} S_{p}\left[\left(P_{b}+P_{0}\right)-\left(P_{a}+P_{0}\right)\right]$

where $C_{f}$ is a force coefficient changing with the valve's lift, to take account of the pressure drop across the valve ${ }^{[7,8]}$, Sp is the pressure area, $\left(P_{b}+P_{0}\right)$ the cylinder pressure, and $\left(P_{a}+P_{0}\right)$ is the pressure in the respective inlet plenum.

\section{SPEED ANALYSIS OF THE VALVE}

The state of the suction valve and discharge valve will be analyzed. The suction valve will be closed at the state of compression and discharge. And with the piston rebound the compressor begins to expand, so the inner pressure decreases little by little. With the end of the inflation the outer pressure of the cylinder which may be outer-air or buffer will conquer the pressure of the spring and the medium will plunge into the cylinder when the suction valve opened, then the suction state begins. The equations of the suction and discharge valve will be established according to the theory of the valve motivation. Such as suction valve in the whole process will pass through that the piston reverses to expands, suction valve begins to open, the piston moves to the inner deadline, the suction valve 
is closed when the piston returns, the inner pressure begins to be established, discharge valve is opened and gas discharges. All of the process can be divided into eight phases, and the motion of the suction and discharge valves will be separately thought from the whole process:

\section{A. Periods of Discharge Valve Closed to the Suction Valve Prepare to Open}

The piston can think as rebound back from far point according to the internal gas pressure and at the time the discharge valve has closed and suction valve prepares to open. After the discharge valve closed and the piston continues to return, the gas pressure in the cylinder begins to decrease with inflation. The suction valve will be prepared to open when the pressure decreases to pressure difference of the inner-outer higher above the spring force of the valve. And the pressure decreasing and valve motion mainly has relation with the moving speed of the piston, the characters of the gas and every clearance volume.

\section{1) The ways get the pressure in the cylinder:}

a) First: The pressure probes were put directly on the cylinder whose advantage is direct and accurate, but whose shortcoming is difficult installtion, especially more difficult to the compressor has been finished. The pressure and vibration curve is shown in the figure 4.

b) Second: The pressure can be calculated according to the vibration data on the outer cylinder through analyzed the relation between vibrations and pressure which changes according to the time. The trend is shown in the figure 5 . Contrast to the above way it's easier to get the vibration data on the surface. Whose shortcoming is the pressure curve only expresses trend but not the accurate data.

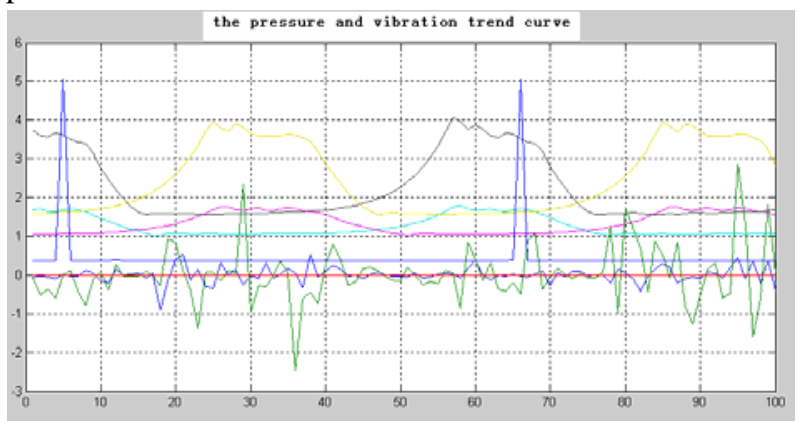

Figure 4. The vibration and pressure curve of the cylinder

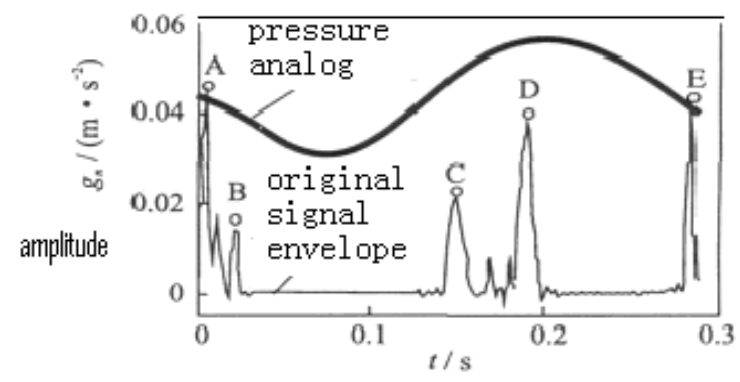

Figure 5. The analog pressure curve
According to the vibration of the surface the changes of the pressure in the cylinder can be calculated through motive equations which need to think about every boundary conditions. The $t_{0}$ can be supposed to the beginning time when the discharge valve closed and the pressure difference between the discharge pressure and cylinder is equal to the spring force. We can get the next equations through analyze every force condition and get the time $t_{1}$.

$$
\begin{aligned}
& d p+k p \frac{d V}{V}-k p_{s}\left(\frac{p}{p_{s}}\right)^{\frac{1}{k}} \frac{\alpha_{S V} A_{S V}}{V} \cdot \sqrt{2 \frac{K}{K-1} R T_{a}\left[1-\left(\frac{p}{p_{s}}\right)^{\frac{k-1}{k}}\right]} d t=0 \\
& \frac{d^{2} y}{d t^{2}}+\frac{a^{2}\left(k_{1} L_{1}\right)^{4}}{L_{1}^{4}} y=\frac{1.566}{A_{c} \rho} \beta b\left(p_{s}-p\right)
\end{aligned}
$$

\section{B. The Open Time of the Suction Valve}

The process is the suction valve is opened at unsteady acceleration. Think about calculate the changes of the gas force with boundary conditions when the time is 0 , the valve is closed and the pressure difference is equal to the spring force. Then at last when the valve was opened entirely the pressure inner cylinder decreases the least and the pressure difference increases the most. We can get the pressure from the figure 4 or 5 , and then accumulate the pressure to the area and get the force. The spring force and the pressure difference have a proportion with the length of the spring. The accumulation length is the distance between close and open entirely of the valve. The quality of the valve is $\mathrm{M}$. According to the formula 11,12 , combined with the valve acceleration motive equation we can calculate the active time $t_{2}$. It must need some time at the process of the valve opened without the time the valve won't open and the gas won't suck into the cylinder. Usually the time of the valve open is about the $1 / 20 \sim 1 / 10$ time of the whole processing, about which we can estimate the whole processing time. That is one of the reasons why we can't increase the speed of the compressor infinitely.

\section{Suction Time of the Valve}

The time depends on the piston running time is the periods of the valve open entirely to the preparation of close whose definition is the valve open entirely and the pressure difference is equal to the spring force again. The length of the time according to the speed of the piston, the length of the cylinder and the dead point of the cylinder is main periods in the whole process and actually depends on the angle speed of the crank. The time will be calculated easily. If the angular speed of the crank is $\delta$ then the circle time is $t_{3}=\frac{2 \pi}{\delta_{p_{1}}-\delta_{p_{2}}}$, and the $p_{1}, \quad p_{2}$ express the pressure of the valve open entirely and the pressure that the valve prepares to close, which can be thought to be the same as the time that the valve arrives at the dead point if the precision is not very accurate, which is a little later than the dead one. 


\section{Close Time of the Suction Valve}

When the piston returns from the far point the suction valve begins to close and the pressure arise. The period is the valve unsteady speed up closing time under the changed pressure. The close length $\mathrm{h}$ as figure 3 is the distance between close and open entirely of the valve. The quality of the valve is $M$, the differential pressure $\left(P_{b}-P_{a}\right)$ gets from the pressure changed curve shown in figure 4 and 5 , as the open time of the valve, and with the motive equation we can get the closed time $t_{4}$.

\section{E. Compression Time}

The period is suction valve closed and discharge valve prepared to open. When the suction valve finished close and the piston continue to move, the pressure arises when the gas in the cylinder was compressed continually. When the pressure arises to the pressure difference between the inner and outer is equal to the spring force the suction valve prepares to open and this period is $t_{5}$. The arising pressure trend in this period mainly has the relation with the piston speed, the gas characters and every clearance volume. Especially the open time of the discharge valve has the relation with the spring force and the out pressure of the pipe, which also has affection with the material, if the material viscosity is too high which will make the valve open later. As the part 3.2.1, we can get the time $t_{5}$ through diagnosis the pressure directly, vibration push back and calculate with equation separately.

\section{F. Open time of the Discharge Valve}

The period $t_{6}$ is the discharge valve varied accelerating open process which calculation thinks mainly about the gas force changes with combined boundary conditions, when the time is 0 , the discharge valve is closed, the pressure difference is equal to the spring force, and at last when the discharge open entirely the pressure arises the highest and the pressure difference is the most. According to the equation 11 and 12, we can calculate the running time of the valve combined with the valve acceleration speed equation.

\section{G. Discharge Time}

The discharge valve will open entirely during the period which depends on the piston running time as the suction time. We can suppose that the time is $t_{7}$ which can be calculate from the speed of the piston, the angular speed, the length of the cylinder and the dead point of the cylinder and actually the radius of the crank. If the angular speed of the crank is $\delta$ then

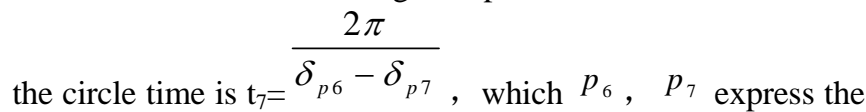
pressure the discharge valve opens entirely and the pressure the discharge valve prepares to close which can be taken as the dead point time as the suction time.

\section{H. Close Time of the Discharge Valve}

The discharge valve will close, gas expand and the pressure begin to decrease when the piston return from the dead point near the cylinder head. The acceleration close time $t_{8}$ on the condition of the variable pressure can get from the motive equation from the pressure derivation.

\section{SYNTHETICAL ANALYSES}

From the above analysis we know that all the time of the valve motive is the sum of the $t_{1} \sim t_{8}$, which affected not only by the running speed of the piston but also by the characters of the gas, the spring force, the inertial force, the speed of the valve, the dead point and so on. And the time suffered the pressure and quantity of the gas that we can't rise up infinitely and the top-speed will suffered by the valve. So the limit ion characters of the reciprocating compressor are limited by the valve speed. We should find other methods to improve the characters of the compressor but not only increasing the speed of the piston speed.

1) Increase the quantity of the valve and increase the size of every valve.

2) Expand the direction of the cylinder.

3) Prolong the distance of the piston.

4) Decrease the dead point.

5) Design the new type valves that can decrease the loss that the gas passes through.

6) Increase the partner accuracy and decrease the machine loss.

\section{CONCLUSIONS}

Through analysis of the motive characters about the valve we can know that the valve needs some time to finish the whole motive process of the inflation, suction valve open, intake, close, pressure, discharge valve open, discharge, close. The process affected by the characters of the valves, so we can't increase the quantity and pressure only through increasing the speed of the crank. The increasing pressure and quantity of the gas can get from the increasing the quantity of the valve, increasing the diameter of the cylinder, increasing the route of travel, but this will expand the size of the compressor which will increasing the cost and affection of the field installation.

\section{REFERENCES}

[1] M. Costagliola, "Theory of spring-loaded valves for reciprocating compressors", Journal of Applied Mechanics-Transactions of the ASME 17 (4) (1950) 415 - 420.

[2] M. Wasmbasganss, "Mathematical modelling and design evaluation of high-speed reciprocating compressors”, Ph.D. Thesis, Purdue University, 1966.

[3] M. Elhaj,etl, "Numerical simulation and experimental study of a twostage reciprocating compressor for condition monitoring” Mechanical Systems and Signal Processing 22 (2008) 374-389.

[4] Zheng Jun, Chen Xiao-kai and Lin Yi, "The analysis of the affection facter of the mechanical model of the air spring and characte,” Journal of Agriculcure and Machinery, 2008.5(39)5

[5] Machu E· H· New developments in the stepless reverse flow, capacity controle systeme for reciprocating compressors, M. Hoerbiger Vantilwerke AG,1994.

[6] Wu Rong-ren, Niu Wei-fei, "The analysis of the adjust discharge gas of pushing up the suction valve of the piston compressor", Fluid Machinery 2003,vol31,NO.7,pp:12-15

[7] S. Rao, Mechanical Vibrations, third ed., Addison-Wesley, Inc., NY, USA, ISBN 0-201-59289-4, 1995.

[8] J. Fleming, A theoretical and experimental investigation of the flow of gas through reciprocating compressor valves, IMech C390/011, 1989, pp. 117-119. 